http://dx.doi.org/10.18359/ravi.2004

\title{
Aprovechamiento de residuos orgánicos agrícolas y forestales en Iberoamérica $^{1}$
}

\author{
Álvaro Chávez Porras² \& Alejandra Rodríguez González \\ Universidad Militar Nueva Granada, Colombia
}

Recibido, febrero 01 de 2016

Concepto evaluación, marzo 17 de 2016

Aceptado, junio 30 de 2016
Referencia: Chávez Porras, A.; Rodríguez González, A. (2016). "Aprovechamiento de residuos orgánicos agrícolas y forestales en Iberoamérica". Revista Academia y Virtualidad, 9, (2), 90-107

\section{Resumen}

El presente trabajo muestra una revisión de la gestión de los residuos sólidos orgánicos de zonas agrícolas y forestales, así como sus alternativas de reutilización la cuales han sido implementadas en diferentes países, forjando alternativas de adaptación sobre los cambios. Las actividades antrópicas han generado desarrollo de la economía y con ello lo que se conoce como consumismo; al día de hoy se presenta como resultado un incremento de residuos o materiales que son descartados. Cuando éstos tienen una gestión y/o disposición inadecuada, se generan efectos nocivos que favorecen los estragos, ayudados con la presencia de fenómenos extremos. Considerando el problema donde el total de los residuos sólidos generados en las zonas urbanas es aprox. 1,3 millones de t/año, con una tasa per cápita de 1,20 kg/día - siendo el 46\% residuos sólidos orgánicos, en algunos sectores como el agrícola y forestal en Iberoamérica, se plantean alternativas de manejo de estos desechos con la metodología de las 3R donde se proyecta reducir, reutilizar y reciclar. Esto con el propósito de mejorar su gestión y crear prácticas de "producción más limpia" a fin de establecer procesos sostenibles que favorezcan el aprovechamiento y mejoren el rendimiento de la producción, especialmente la agrícola.

Palabras clave: residuos sólidos orgánicos, residuos agrícolas, residuos forestales, las 3R.

\footnotetext{
${ }^{1}$ Artículo de investigación científica y tecnológica, resultado del proyecto de investigación derivado del proyecto ING 1652, financiado por la Vicerrectoría de Investigaciones de la UMNG, Vigencia 2014-2015.

${ }^{2}$ Ingeniero Industrial, Universidad del Valle, Cali, Colombia; Doctor en Ingeniería Civil, Área Ambiental, UNICAMP, Campinas, Brasil; Líder Grupo PIT, Universidad Militar Nueva Granada, Bogotá D.C. Colombia. alvaro.chavez@unimilitar.edu.co ${ }^{3}$ Bióloga, Universidad Militar Nueva Granada; Asistente de Investigación, Facultad de Ingeniería, UMNG,
Bogotá, Colombia. mandie.a@gmail.com.
} 


\title{
Harnessing agricultural and forestry organic waste in Latin America
}

\begin{abstract}
This paper offers a review of the management related to organic solid waste derived from agriculture and forestry as well as other options, i.e. the reuse concept implemented in different countries, generating adaptation options to changes. Anthropic activities have generated a virtual economic development resulting the so-called consumption; nowadays, it is evident a waste or discarded material increased. An inappropriate management and/or disposal might produce harmful effects sometimes helped by extreme events. Considering the issue where the total solid waste in urban areas is $1.3 \mathrm{MTY}$ approx. with $1.20 \mathrm{~kg} /$ day per capita rate, i.e. $46 \%$ of organic solid wastes, then several sectors, such as agriculture and forestry, in Latin America have proposed different management alternatives following the 3R's methodology in order to plan to reduce, reuse and recycle. All of this to improve their management practices and create "cleaner production" in order to establish sustainable processes that may encourage the use and improving production efficiency, particularly farming.
\end{abstract}

Keywords: organic solid waste, agriculture waste, forestry waste, 3R's.

\section{Aproveitamento de resíduos orgânicos agrícolas e florestais em Ibero América}

\section{Resumo}

O presente estudo mostra uma revisão da gestão dos resíduos sólidos orgânicos de zonas agrícolas e florestais, igualmente suas alternativas de reutilização que têm sido praticadas em diferentes países, criando alternativas de adaptação sobre as mudanças. As atividades antrópicas têm gerado desenvolvimento da economia e no mesmo tempo o que conhecemos como consumismo; até o dia de hoje apresentasse como resultado um incremento de resíduos ou materiais que são descartados. Quando esses têm uma gestão e/ou disposição inadequada, geram-se efeitos nocivos favorecendo os estragos, ajudados com a presença de fenômenos extremos. Considerando o problema onde o total dos resíduos sólidos gerados nas zonas urbanas é aprox. 1,3 milhões de t/ano, com uma taxa per capita de 1,20 kg/dia -sendo o 46\% resíduos sólidos orgânicos, em alguns setores como o agrícola e florestal em Ibero américa propõem-se alternativas de manipulação desses desperdícios com a metodologia das $3 \mathrm{R}$ onde se idealiza reduzir, reutilizar e recicla-los. Isso com a intensão de melhorar sua gestão e criar práticas de "produção mais limpa" com finalidade de estabelecer processos sustentáveis que favoreçam o aproveitamento e melhorem o rendimento da produção, especialmente agrícola.

Palavras chave: resíduos sólidos orgânicos, resíduos agrícolas, resíduos florestais. As 3R. 


\section{Introducción}

El incremento de las áreas o zonas urbanas, así como su población residente, ha desarrollado de forma paralela la producción, consumo de recursos, transporte y en general el comercio; llevando de la mano mayor generación de residuos sólidos y líquidos, lo que afecta el nivel de vida en algunos países generando una problemática social de consumo y demanda, tanto de productos como de servicios (Facua, 2009; Steffen et al., 2004; Programa de las Naciones Unidas para el Medio Ambiente-PNUMA, 2002).

Para 2012, se estimó que las ciudades produjeron cerca de 1,3 millones de t/año de residuos sólidos (RS), con una producción per cápita de 1,2 kg/día. Suponiendo un incremento de residentes urbanos para el 2025 en 4,3 millones, se estima un aumento de RS, hasta alcanzar los 2,2 millones de t/año, con valores per cápita de 1,42 kg/día. (Hoornweg y Bhada-Tata, 2012).

A nivel mundial, cerca del $46 \%$ del total de RS son residuos sólidos orgánicos (RSO), tal como se muestra en la figura 1 (Hoornweg y Bhada-Tata, 2012).

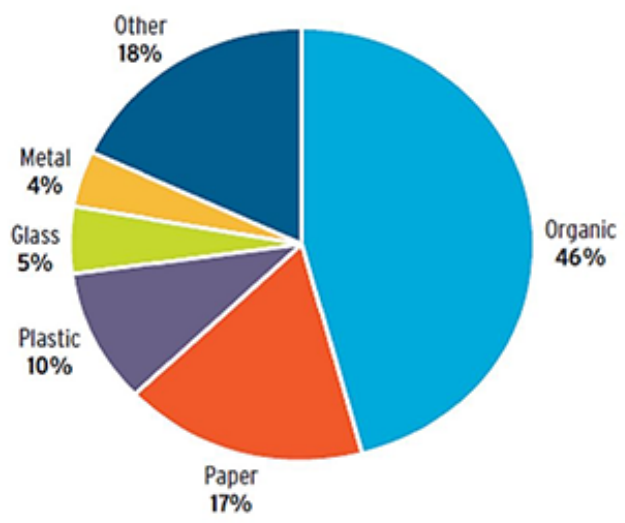

Figura 1. Distribución de la producción - Tipos de residuos a nivel mundial.

Fuente: Hoornweg y Bhada, 2012.

Con el fin de disminuir el impacto ambiental generado por los "fenómenos extremos" que hacen referencia a los cambios atmosféricos y la movilidad del cuadro hídrico a nivel global, los cuales son producidos por las actividades antrópicas que generan eventos naturales intensos. A nivel internacional se han planteado diferentes alternativas de manejo; dentro de ellas se encuentra la metodología de las 3 R. En ésta se propone "reducir, reusar y reciclar" la mayor proporción de materiales usados, en los diferentes procesos de producción; todo con el fin de mejorar la gestión y crear prácticas de "producción más limpia", estableciendo procesos "sostenibles" que favorezcan el aprovechamiento y mejoren el rendimiento económico (Organización para la Cooperación y el Desarrollo Económico [OCDE]. 2010; Frondel, Horbach y Rennings, 2007).

Dentro del $46 \%$ de los RSO, se encuentran aquellos provenientes de actividades domésticas, agrícolas y forestales, dispuestos en rellenos sanitarios (59\%). No obstante, zonas como Norteamérica y Europa han planteado actividades para aplicar las $3 \mathrm{R}$ en sus prácticas de disposición, acompañadas de normativas, multas o "recompensas" a sus ciudadanos, con el fin de hacer cumplir estas medidas (Hoornweg y Bhada-Tata, 2012; OCDE, 2010).

Dentro de las alternativas de reducir, se 
encuentra el rediseño de productos o sistemas de producción, fabricación y consumo, para disminuir las emisiones de gases efecto invernadero (GEI). En cuanto a la reutilización $\mathrm{y}$ al reciclaje, se pretende recuperarlos, de tal forma que sean incluidos de nuevo en la cadena productiva. (Facua, 2009; Frondel, Horbach y Rennings, 2007; Organization for Economic Co-operation and DevelopmentOECD, 2011).

En este artículo se presenta una revisión sobre el uso alternativo de RSO, provenientes de actividades agrícolas $\mathrm{y}$ forestales. Aprovechados con diversas alternativas "ambientalmente correctas", lo que genera opciones sostenibles que favorecen la disminución de efectos nocivos al medio. De igual forma, se presentan alternativas para aumentarles la vida útil, dándoles valor agregado; sólo disponiendo de aquello que no puede ser aprovechado.

\section{Marco de referencia}

A continuación se presentan antecedentes en cuanto al manejo de RS a nivel mundial y cómo se llevan debido a los cambios de las interacciones físicas, químicas y biológicas, generando sobre el planeta lo que se conoce como el cambio ambiental global (CAG); dentro del cual se encuentran los "fenómenos extremos", que significan la asimilación de cambios atmosféricos y la movilidad del cuadro hídrico del planeta (IAI, 2005).

Según la Organización Meteorológica Mundial (OMM-ONU), estos "fenómenos extremos" o "climáticos extremos" están relacionados con la evolución del clima, originario del CAG”. En el 2013 se presentaron impactos desmedidos de sequías, olas de calor, inundaciones y ciclones tropicales, que junto con 2007- fueron años con registros de picos de altas temperaturas, lo que en general produjo precipitaciones copiosas, calores agudos, tormentas acrecentadas, inundaciones en los litorales y aumento del nivel del mar. (Potsdam Institute for Climate Impact Research y Climate Analytics-PICIRCA, 2013; UN, 2009; Bates et al., 2008). Asimismo, se plantea información sobre la posibilidad del uso de RSO, dándoles un papel como posibles fuentes de energía.

\section{Antecedentes en el manejo de RS}

La población se ha incrementado a nivel mundial en 7.000 millones de habitantes, según la Organización de las Naciones Unidas (ONU). Considerando que para el siglo $\mathrm{X}$ apenas llegaba a los 300 millones, para el 2014 se tiene un estimado de 7.300 millones, donde América Latina y el Caribe no escapan a la situación, con un promedio a este año de 606.000.000, pues la población urbana presenta un porcentaje mayor a la media mundial, la proyección para el año 2050 muestra que el $68,7 \%$ de la población mundial se encontrará en áreas urbanas (Comisión Económica para América Latina y el Caribe-CEPAL, 2013; Crossette, 2011; Tello, et al., 2011; Bogner, et al., 2007).

La vida social y las relaciones como urbe crearon un impulso industrial y agrícola, generando un permanente aumento en la cantidad de desechos por disponer. Éstos existen desde hace 4.000 millones de años, donde en tiempos pasados su eliminación no representaba un problema específico, por ser una población pequeña y las áreas de depuración natural, extensas. La problemática da inicio con el desarrollo de la sociedad moderna, por las cantidades generadas y la difícil asimilación natural, debido a la calidad de los mismos, lo que dificulta la gestión año tras año. (Population Reference Bureau-PRB, 2013; Crossette, 2011; Bogner, et al., 2007).

Todos estos factores de sobrepoblación, actividades antrópicas y consumismo, han ayudado a almacenarlos en grandes cantidades, generando perspectivas de gestión y tecnologías adecuadas para reciclarlos. No obstante, mientras se continúe con quemas no controladas y disposición en tiraderos a cielo abierto, seguirán presentándose problemas de contaminación, enfermedades y vectores (Tello et al., 2011; Facua, 2009).

La incineración de residuos sin clasificar 
donde se encuentran plásticos, pilas, baterías, entre otros, produce sustancias como dioxinas y furanos, elementos tóxicos y cancerígenos. Los principales efectos de la disposición inadecuada incluyen la degradación de materia orgánica en gases como metano y dióxido de carbono (gases de efecto invernadero), presencia de contaminantes en el suelo, que afectan sus propiedades, contaminación de fuentes de agua superficial y subterránea, finalmente efectos sobre flora $y$ fauna circundante a los sitios de disposición (Tello et al., 2011).

Teniendo en cuenta que se hace referencia como "residuo" a un elemento que es desechado, dispuesto o que requiere de disposición final, de acuerdo a las normativas establecidas por cada país. En general, es un elemento denominado "inservible" ya que no tiene un propósito o se ha obtenido como excedente en el proceso de producción; una vez que ha cumplido su objetivo, como en el caso de los empaques; asimismo, en caso de no ser usado con fecha límite de uso. (Decreto 4741, 2005; Instituto Colombiano de Normas Técnicas y Certificación-ICONTEC. 2009; Pongrácz y Pohjola, 2002; Pongrácz y Pohjola, 2004; OECD, 2000).

Actualmente, una de las alternativas que contribuye a disminuir el impacto que generan es la tecnología end of pipe, la cual consiste en realizar un manejo al final del proceso productivo. Una forma es la incineración, metodología costosa, que requiere instalaciones específicas para mantener los residuos secos; además, genera polución y cenizas que necesitan control de partículas a la salida. La otra opción, la más implementada, son los rellenos sanitarios; donde las emisiones líquidas (lixiviados) y gaseosas (gas metano) son sometidas a diversos procesos de tratamiento o aprovechamiento a fin de disminuir los efectos generados al medio natural (Hoornweg y Bhada-Tata, 2012; Arrayave y Garcés, 2007; Arroyave y Garcés, 2006).
Esta disminución de residuos y las consideraciones de manejo hasta su destino final, generan lo que se define como "producción más limpia-PML". Uno de los ejemplos es el manejo de los RSO procedentes de la industria agrícola y forestal, los cuales se presentan como un potencial de vida, ya que son biodegradables, lo que posibilita una recuperación natural de sus "propiedades nutritivas" a través de la estabilización química. Aunque en varios países todavía se consideran una problemática socioambiental, en otros, se plantea el camino de cómo reducir, reusar y reciclar a través de los programas de PML y desviarlos de los sitios de disposición final, para ser tratados y aprovechados, minimizando también la necesidad de los rellenos sanitarios (HonduPalma, 2011; OCDE, 2010; Guajardo, 2010).

Según el Programa de Naciones Unidas para el Medio Ambiente-PNUMA (2013), este avance se está generando mediante la integración de áreas dedicadas a temas específicos, tales como las estrategias de una producción sostenible; la cultura de reducción de desperdicios en los procesos; la adecuada separación en la fuente; la preparación del modelo de reciclaje; el aprovechamiento final y minimización de la disposición en el relleno; la gestión integral de residuos especiales y peligrosos; entre otros. Cabe destacar que la reutilización presenta contrariedades con la definición. Esto implica que a pesar de haber cumplido su propósito, el estado y las características del elemento no lo hacen inútil, pero puede ser reintegrado dentro de la cadena productiva; por lo cual no sería considerado como residuo. (HonduPalma, 2011; Pongrácz y Pohjola, 2002; Pongrácz y Pohjola, 2004).

En general, el concepto de residuo, tal como se muestra en la figura 2, debe presentar el ciclo genérico de la operación, donde se muestra un manejo, su propósito, la estructura o proceso, el protocolo de acción y la realización o resultados (Pongrácz y Pohjola, 2004). 


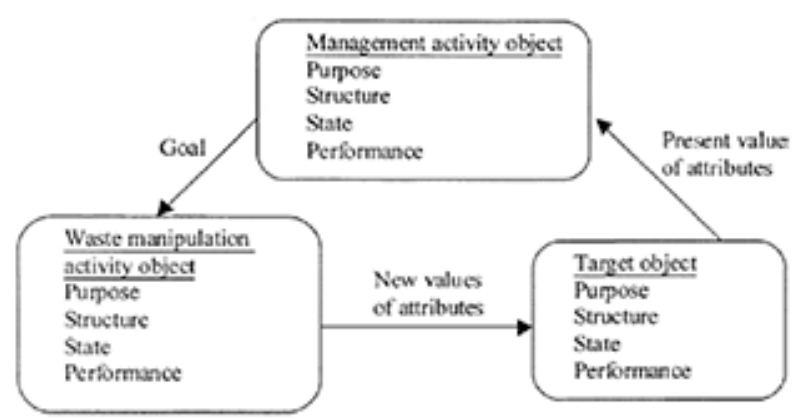

Figura 2. Ciclo genérico del manejo de residuos. Fuente: Pongrácz y Pohjola, 2004.

No obstante, existen aclaraciones, tal como se encuentra en la normativa colombiana, en la cual se hace alusión a los "residuos aprovechables", como cualquier material, objeto, sustancia o elemento que no tiene valor de uso directo o indirecto para quien lo genere, pero susceptible de incorporación a un proceso productivo de otro (Decreto 1713, 2002). La producción de residuos depende, en gran medida, de las condiciones que presenta cada país; relacionado con el ingreso promedio, al producto interno bruto (PIB) y al consumo de la población, ya que el acceso a recursos influye en cuánto, cómo y qué se consume. Por ejemplo, en la zona de África Subsahariana se obtiene una producción anual de 62 millones de t/año, con un rango per cápita de 0,09 a 3,00 kg/día; en aquellas ciudades donde hay mayor desarrollo de turismo, se tiene promedio más alto. Para el caso del Este de Asia y la región del Pacífico que incluye a China, se producen cerca de 270 millones de t/año, con un rango per cápita de 0,44 a $4,30 \mathrm{~kg} /$ día. Mientras que en Latinoamérica y el Caribe, sólo se generan 160 toneladas por año y la tasa per cápita está entre 0,10 a 14,00 $\mathrm{kg} /$ día; y los valores más altos se presentan en las islas, debido al comercio que se desarrolla (Hoornweg y Bhada-Tata, 2012).

En general, aquellos países con ingresos bajos adquieren menos cantidad de materiales procesados; principalmente usan elementos provenientes de la agricultura. Por su parte, los de alto ingreso denominados "desarrollados", tales como Bélgica, Canadá, Francia,
Alemania, Japón, Estados Unidos, entre otros, generan mayor cantidad de residuos de papel (31\%), debido al desarrollo de la industria, comercio, actividades en oficinas y hogares (revistas, folletos, periódicos, empaques, etc.); considerando que la producción de plásticos, vidrio, metales y otros corresponde al acceso a productos procesados y empacados por la industria (FAO, 2013)

En cuanto a aquellos de ingresos medios, presentan un punto intermedio, para todas las categorías, ya que tienen acceso a una gran cantidad de productos, tanto empacados como procesados y naturales. No obstante, cabe resaltar que estos últimos ocupan gran parte de la actividad económica que se desarrolla en torno al sector agrícola. Entre estos países se encuentran Ecuador, India, Paraguay, Colombia, México, Panamá, entre otros. (FAO, 2013; Hoornweg y Bhada, 2012).

\section{Residuos orgánicos como fuente de energía}

Los residuos en general están considerados por la legislación colombiana, en el Decreto 4741 de 2005, como "cualquier objeto, material, sustancia, elemento o producto que se genere en estado sólido, semisólido, o líquido o gas contenido en recipientes o depósitos, cuyo generador descarta, rechaza o entrega porque sus propiedades no permiten usarlo nuevamente en la actividad que lo generó o porque la legislación vigente así lo estipula" (Ministerio de Ambiente, Vivienda y Desarrollo Territorial-MAVDT, 2005). 
Dentro de estos se encuentran los RS y con ellos los producidos en municipios o residuos sólidos municipales (RSM), los cuales están compuestos de ítems de uso diario como plásticos, empaques, césped, muebles, ropa, botellas, restos de comida, periódicos, electrodomésticos, pintura y baterías; según la Agencia de Protección Ambiental (EPA, por sus siglas en inglés), estos residuos presentan una generación total (por material) para 2012, correspondiente a 251 millones de toneladas (antes de reciclaje) como lo muestra la figura 3; con una tasa de generación para 1960 a 2012 respectiva, mostrada en la figura 4 (EPA, 2014).

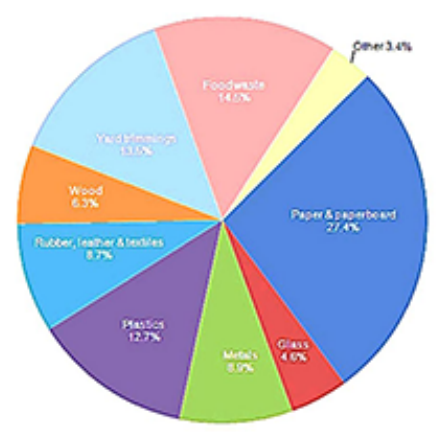

Figura 3. Generación de RSM total (por material) para 2012, correspondiente a 251 millones de toneladas (antes de reciclaje). Fuente: EPA, 2014.

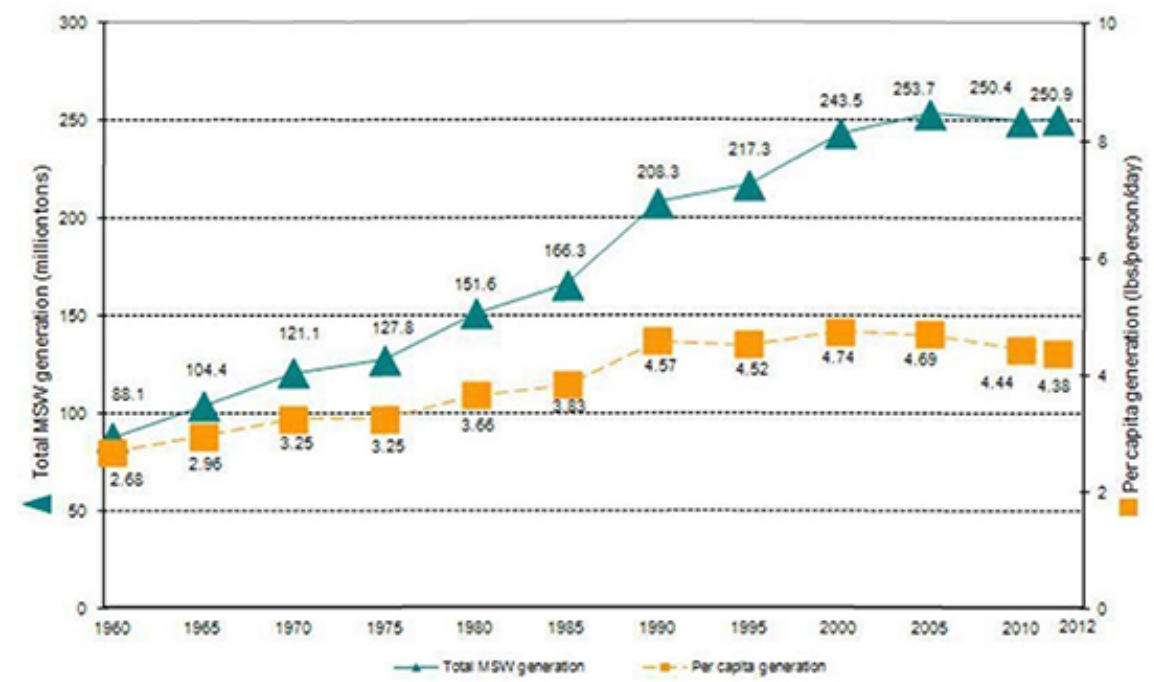

Figura 4. Tasa de generación de RSM de 1960 a 2012. Fuente: EPA, 2014.

Por otra parte, los RS - de acuerdo a sus propiedades- se pueden clasificar en diferentes tipos y de esta forma se proponen las alternativas de reciclaje, eliminación o disposición. En cuanto a aquellos de tipo orgánico (RSO), hacen referencia a aquellos sólidos que se originan naturalmente durante el ciclo vital, como consecuencia de las funciones fisiológicas y/o de mantenimiento; o son producto de la explotación antrópica de los recursos bióticos, que tienen su origen en los seres vivos, animales o vegetales (Sztern y Pravia, 2001).

Debido a su constitución, se plantean alternativas de reciclaje y compostaje, las cuales permiten 
tratar cerca de 86,6 millones de t/año. Lo anterior, desfavorece la liberación de 168 millones de toneladas métricas de equivalente de dióxido de carbono a la atmósfera; es decir, se generan efectos similares de retirar 33 millones de automóviles de las vías durante un año. Por ello, cada país plantea opciones para el manejo de RSO, originados a partir de actividades forestales y agrícolas, con el fin de disminuir el costo de disposición final y disminución del impacto ambiental, que generan cuando no se hace un manejo adecuado (EPA, 2014).

Por eso, hoy los países buscan, con los avances tecnológicos, satisfacer la necesidad para la adecuada disposición o reutilización de los RSM (UAESP, 2010). En el caso de Europa, específicamente España, los generados en explotaciones agrícolas corresponden a un $46 \%$ del total de los RS, con una producción aproximada de 27.000 .000 t/año. De igual forma se obtiene el $40 \%$ de residuos como plásticos, que son aprovechados en puntos de acopio y reciclaje (Ministerio de Agricultura, Alimentación y Medio Ambiente, MAAMA, 2012).

Para Latinoamérica, específicamente en cultivos agrícolas de Colombia, los desechos encontrados de la ciudad de Medellín, identifican que solo 20 al $30 \%$ son aprovechables; por tanto, existe la necesidad de promover actividades viables para hacer de esta capital una mejor urbe del futuro; ya que con sistemas de manejo eficientes se logra disminuir el fruto de los gases emitidos $\mathrm{y}$ el efecto nocivo que genera la inadecuada gestión de los mismos.

\section{Estudios de caso. Residuos agrícolas}

En las actividades agrícolas de varios países no se realiza la disposición de residuos en un relleno o zona adecuada, siendo la opción más económica la quema descontrolada del material. Esto permite al agricultor reducir el volumen de material, limpiar, despejar la zona para el cultivo, eliminar plagas y liberar nutrientes (CCA, 2014; Magrama, 2013). No obstante, genera efectos nocivos al medio ambiente la quema de biomasa como madera, hojas y árboles, originando de su total $40 \%$ en $\mathrm{CO} 2,32 \%$ en $\mathrm{CO}, 20 \%$ en material particulado y $50 \%$ en hidrocarburos aromáticos policlílicos (HAP), emitidos a escala mundial (CCA, 2014). Vale resaltar que a nivel europeo, los residuos agrícolas, forestales y acuícolas corresponden al 2\% del total del RS (CEPAL, FAO e IICA, 2014; Eurostat, 2014).

En general, productos que intervienen en el producto interno bruto (PIB) de los países, como el cereal, generan entre 1,4-4,3 t/h de $\mathrm{RSO}$, teniendo en cuenta que existen zonas donde se hace un aprovechamiento para alimentación del ganado. En los cultivos industriales como el algodón o semillas oleaginosas, no se presenta ningún tipo de aprovechamiento, generando un promedio de 1,0-10,0 t/ha, que son eliminados por incineración. Para los cultivos destinados a frutas y viñedos, donde se realiza una poda anual, se producen en promedio de 1,7-5,33 t/ ha, de acuerdo al tipo de planta, que en muchos casos también es incinerado (EIA, 2008).

Dentro del Catálogo Europeo de Residuos (CER) [MAM/302/2002], se incluyen como residuos, los materiales plásticos usados en las actividades agrícolas. Desde 1960, se ha planteado el uso de éstos, como alternativa a los invernaderos de vidrio. En las actividades actuales, se usan acolchados de plástico negro para evitar el crecimiento de malezas o plantas no deseadas que puedan competir con el cultivo, lo que favorece la disminución de tiempo para la cosecha y la necesidad de instalar tuberías plásticas que permiten un uso eficiente del agua y fertilizantes, entre otros. A pesar de sus ventajas, en las actividades de cosecha se generan daños y rompimientos, creando una gran cantidad de desechos que presentan un problema en cuanto a recolección y disposición (Macua, et al., 2009).

Según análisis realizados, estos residuos constituyen cerca del 5\% en el sector agrícola. 
Dentro de estos materiales plásticos se usan los de diferentes calibres, desde 100 um hasta 200, de acuerdo a la actividad en la que estén involucrados y los objetivos del agricultor. Una vez usados, las alternativas de manejo más aplicadas constan de incineración controlada o abandono en zonas no aptas para su disposición, forjando conflictos ambientales como emisión de monóxido de carbono y liberación de elementos pesados, derivados del uso de componentes químicos (Macua et al., 2009).

En el caso de España, no se tiene un promedio general de todos los residuos agrícolas, sin embargo; en una estimación independiente para el 2007 se tiene un promedio de 343 millones t/año en residuos agrícolas, de las cuales 27 millones aprox. corresponden a residuos de cultivos; dentro de éstos, 32.7\% corresponden a cultivos de cereales; su gestión se encuentra consignada en la Ley 10/1998. (Magrama, 2012; Cuadros, 2008).

El área de Andalucía cuenta con una superficie de 3.674.00 ha, donde se ubican 8 zonas de alta concentración de actividad agrícola. En una de ellas, Almería, se encuentra la del Ejido, conocido como el "mar de plástico", que ocupa 30.000 ha aproximadamente. De acuerdo a datos obtenidos en el Plan Director Territorial de Gestión de Residuos Urbanos en Andalucía se obtienen cerca de 42.000 t/ año de residuos plásticos, donde 21.600 $\mathrm{t}$ corresponden a acolchados (polietileno normal) y $20.800 \mathrm{t}$ a invernaderos (polietileno de larga duración); el restante se compone de los túneles de cultivo, tuberías para riego y materiales de envases y embalaje. (Macua et al., 2009; EOI, 2008).

Debido a que esta zona tiene la mayor cantidad de residuos plásticos del sector agrícola, se han creado centros de aprovechamiento desde 1991. El problema más común es el nivel de contaminación del material por partículas de tierra, arena, vegetales y otros, que se encuentran en el cultivo, dificultando el reciclado. Asimismo, el $40 \%$ de los plásticos provenientes de invernaderos no son dispuestos adecuadamente, lo cual incrementa su volumen, haciendo que el agricultor los deseche en cualquier sitio o proceda a incinerarlos (Macua et al., 2009).

Actualmente los programas de mayor alcance para la gestión de estos residuos son: Sigfito, con el cual se busca recolectar y tratar los envases de productos fitosanitarios; y CicloAgro para la recolección y tratamiento de residuos plásticos (Magrama, 2012). El primero es una sociedad sin ánimo de lucro que busca hacer una gestión adecuada a los envases agrícolas, con el apoyo de los fabricantes y envasadores de productos fitosanitarios; el proceso se muestra en la figura 5. Al agricultor se le ofrecen puntos de recolección, en los que se obtiene un certificado de buena disposición, evitando multas o sanciones. Además, en convenio con las empresas fabricantes, la mayoría de los empaques que son recibidos por este programa llevan el logo de Sigfito, facilitando su identificación; de igual forma se tiene un sistema de premios a los puntos de recolección, lo que favorece las buenas prácticas (Sigfito, 2012).

Dentro de las alternativas se encuentran el reciclado mecánico, que los reincorpora a la cadena productiva para la producción de bolsas, contenedores, mobiliarios, entre otros. Para este proceso se requiere una materia prima libre de contaminantes y con poco desgaste, el cual es recogido, seleccionado y triturado hasta el tamaño de una lenteja, denominado "granza"; esto, para facilitar la transformación por la acción de calor y presión hasta obtener el nuevo producto deseado, con una capacidad de tratamiento de 8.300 t/año (Macua et al., 2009). 


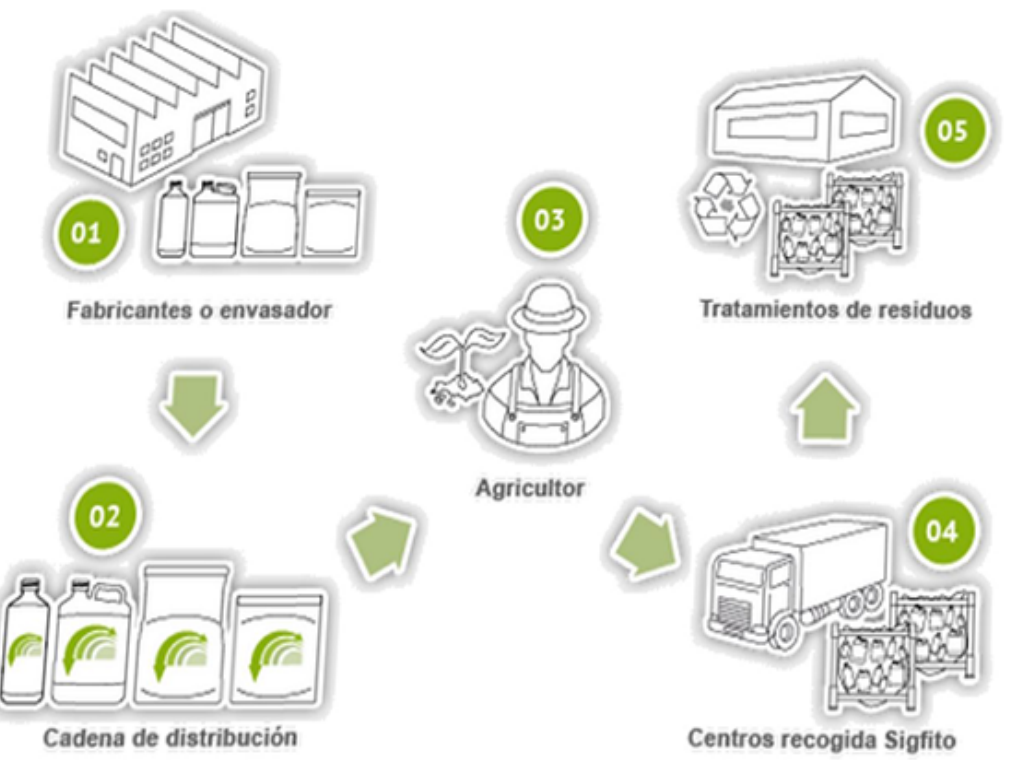

Figura 5. Programa Sigfito. Cadena donde se involucra el material con contenido fitosanitario. Fuente: Sigfito, 2012

La otra opción corresponde a la valorización energética, ya que se pueden usar como combustible, debido a su poder calorífico, tal como se observa en la tabla 1. Con esta alternativa se puede hacer uso del material deteriorado o sucio, lo que ha permitido sustituir el carbón como combustible en las centrales térmicas, además, de mantener o disminuir las emisiones al medioambiente. En España, el Decreto 104/2000 valoriza los residuos, incluyendo plásticos (Consejería de Medio Ambiente, 2012).

Tabla 1. Poder calorífico de plásticos y algunos combustibles.

Poder calorifico en $\mathrm{kJ} / \mathrm{kg}$

\begin{tabular}{l|c|}
\hline Poliestireno & 46.000 \\
\cline { 2 - 2 } Polietileno & 46.000 \\
\cline { 2 - 2 } Polipropileno & 44.000 \\
\cline { 2 - 2 } PVC & 18.900 \\
\cline { 2 - 2 } Gas natural & 48.000 \\
\cline { 2 - 2 } Fuel-oil & 44.000 \\
\cline { 2 - 2 } Hulla & 29.000 \\
\cline { 2 - 2 } Lignito & 20.000 \\
\cline { 2 - 2 } Cuero & 18.900 \\
\cline { 2 - 2 } Papel & 16.800 \\
\cline { 2 - 2 } Madera & 16.000 \\
\hline Grasa & 7.800 \\
\cline { 2 - 2 } Basura doméstica & 8.000 \\
\cline { 2 - 2 } Fute: Consejería de Medio Ambiente, 2012.
\end{tabular}


Finalmente, se tiene la opción de reciclarlos químicamente, descomponiéndolos a través de un proceso químico, en componentes más sencillos que permiten su reutilización. Cabe recordar que dentro de estos métodos se encuentran: la pirolisis (descomposición térmica anaerobia entre 400 a $800^{\circ} \mathrm{C}$ ); y la hidrogenación (proceso del $\mathrm{H}$, en condiciones anaerobias y altas temperaturas). En el caso de Navarra, con respecto a la distribución de residuos plásticos se han desarrollado sistemas de recolección por algunas comunidades en puntos específicos para facilitar su transporte, el $69 \%$ de los residuos no cuenta con disposición adecuada, por lo que son enterrados o incinerados. (Departamento de Desarrollo Rural y Medio Ambiente. 2010, 2010).

Mientras que en América Latina los envases residuales de los agroquímicos usados, Argentina ha diseñado alternativas para reciclaje, minimizar el residuo líquido y la opción de crear nuevos envases. De acuerdo a datos registrado para el 1996 se utilizaron cerca de 13.210.000 envases de plaguicidas en el país y 2.710.000 de embalajes. De ellos, $77 \%$ fue de material plástico $(0.44 / \mathrm{h})$, los cuales son llevados a dos centros de aprovechamiento, para ser reciclados como materia prima de otros plásticos. El material reciclado se compone de $50 \%$ de PET y $50 \%$ de envases de agroquímicos. El costo del proceso es de 600 US\$/ton, donde un $50 \%$ cubre la recuperación para la venta de postes y varillas (Allevato y Pórfido, 2002).

No obstante, Brasil a 2001 ya reciclaba el 20\% de los envases plásticos por la ley Federal 9974/00 y el Decreto reglamentario 3550/00, para 2013 se alcanzó el 59\% de envases de tereftalato de polietileno reciclados, correspondiente a 331.000 toneladas. Los residuos son clasificados por color, lavados, triturados, para la creación de nuevos elementos, que luego se someten a peletizados por condensación en frío y finalmente moldeados (Allevato y Pórfido, 2002).

En Colombia, cuando se tenía una superficie bajo cultivo de 30.270 .000 ha, en el mercado se encontraban cerca de 13.200.000 envases por año. Aunque se planteó una recuperación mediante aprovechamiento energético, el gobierno solo ha planteado el desarrollo de centros de acopio y tercerizado las acciones de aprovechamiento (Allevato y Pórfido, 2002).

\section{Residuos de ganadería y tenencia de animales}

Se considera como residuos de ganadería y tenencia de animales aquellos obtenidos en las explotaciones ganaderas de producción y reproducción. Debido a que el estiércol se puede fermentar y usar como abono en plantas, en algunos países no se considera un residuo ni presenta efectos nocivos al medio ambiente, siempre y cuando no se considere una explotación intensiva donde estos sean retirados por presión de agua (EOI, 2008).

En España es mayor la proporción de residuos de subproductos animales no destinados a consumo humano (Sandach, 2015; Magrama, 2012). Las deyecciones varían según la especie; se estima una producción anual de 130 millones de t/año entre estiércoles y purines; siendo el mayor aporte del sector vacuno y porcino con $50 \%$ y $40 \%$. De acuerdo a su composición se tiene diversas alternativas de gestión, entre ellas el uso como fertilizante sin tratamiento, según lo establecido por el Decreto Real 261/1991.

Para el caso de Perú, se tienen registros de los residuos provenientes de ganadería vacuna, ovina, porcina y el sector avícola; pudiéndose identificar en el caso de los vacunos, que $16 \%$ del total de la producción corresponde a residuos, que incluyen subproductos como sangre, pezuña, cachos y excrementos; mientras 51\% pertenece a los cortes de carne. Para los ovinos, $10 \%$ son residuos que incluyen las partes nombradas anteriormente; mientras que $40 \%$ corresponde a carne aprovechable. En el caso de los porcinos, el porcentaje de residuos es $10 \%$, teniendo en cuenta que solo se desecha lo relacionado 
con la sangre y los excrementos, que en algún momento pueden ser aprovechados; lo que demuestra que el sector porcícola tiene un mayor porcentaje de aprovechamiento de los animales. No obstante, la producción avícola, tiene un comportamiento contrario al anterior, ya que se tiene el $18 \%$ de residuos, incluyendo sangre, plumas, buche, cloaca y grasa (Oficina de Estudios Económicos y EstadísticosOEEE, 2011).

\section{Residuos vegetales}

Los residuos vegetales son los que más se encuentran en la producción agrícola, ya que corresponden a las podas de plantas; la extracción de las mismas cuando la cosecha sólo tiene en cuenta los frutos o partes de la planta; frutos que sufren daños y no se pueden comercializar; desyerbe; y otro tipo de actividad complementaria al desarrollo del cultivo que genere eliminación de plantas. Teniendo en cuenta que estos residuos son orgánicos, las alternativas más usadas para su aprovechamiento son el compostaje y el vermicompostaje (Unidad Administrativa Especial de Servicios Públicos-UAESP, 2010; MMAA, 2010).

Colombia muestra cultivos de plátano que cuentan con una participación en el mercado de América Latina de 38,1\% y 9,7\%, respectivamente, del mercado mundial. Anualmente se exportan cerca de 54\% a Estados Unidos, lo que equivale a 117.123 toneladas de fruta fresca y 4.395 toneladas de fruta congelada. A nivel general, se cuenta con una producción anual de 2,9 millones de toneladas de racimos, aunque la mayor parte se utiliza para abastecer el mercado nacional, ya que se cuenta con un consumo per cápita de $62 \mathrm{~kg}$. En el cultivo, solo el racimo de banano es la parte aprovechable, lo cual corresponde a $20 \%$ o $30 \%$ de la planta; mientras el 70 $80 \%$ de ésta se considera residuo. Se han desarrollado métodos para usar el pseudotallo y el raquis como harina en la producción de alimentos, pero no existe una metodología, ni política que lo regule (Mazzeo, et al., 2010;
Espinal, Martínez y Peña, 2005).

En Colombia se comenzó por implementar soluciones de aprovechamiento de residuos agrícolas, debido la falta de recursos con potencial energético como la madera. En Cesar, la comunidad indígena nasa ha desarrollado biodigestores para aprovechar los residuos de las cosechas, con el fin de crear autonomía energética, disminuyendo la tasa de deforestación de la zona, costos por la adquisición de gas propano y conflictos sociales debido al peligro por artefactos explosivos que atentan contra la comunidad (Martínez y Sandoval, 2015).

\section{Residuos forestales}

Los cultivos forestales son aquellas plantaciones donde se realiza la explotación de maderas, que requieren zonas amplias para el crecimiento de árboles. Debido a que son cultivos a largo plazo, son las podas periódicas, raleos y ramas que no se pueden aprovechar, lo que corresponde aproximadamente a un tercio del árbol. Hasta la fecha, los productos forestales se usan como fuente energética e indumentaria a nivel doméstico y comercial. Debido a las propiedades energéticas, el manejo inadecuado de los residuos presentes en el sector, generan efectos nocivos sobre el medio ambiente, ya que favorece la propagación de incendios afectando zonas aledañas naturales y la proliferación de plagas a otros cultivos (Semarnat, 2008; Velázquez Martí, 2006).

En el caso de México se han promulgado leyes para el desarrollo forestal sostenible, donde se plantea el aprovechamiento de los recursos maderables. Ha tenido una producción promedio anual de $5.9 \mathrm{~m} 3$ en rollo para 2013 y recursos no maderables (no leñosos) de 202.789 toneladas, que incluyen hongos, semillas, frutos musgo y heno. Como parte del aprovechamiento, se encuentran la fabricación de tableros y otros materiales requeridos por la industria de la celulosa, cama de ganado, compost y aserrín; este último se puede usar en la cama de corrales o elaboración de papel 
de baja calidad, ya que no se puede incinerar (Secretaría de Gobierno-SEGOB, 2014).

Otro país, como Uruguay, durante la última década ha incrementado la producción anual de madera de $3.153 \mathrm{~m} 3$ en el año 2000 a $14.637 \mathrm{~m} 3$ en 2010 , con fines de aprovechamiento, especialmente como combustible, tal como se ve en la tabla 2.

\begin{tabular}{lc}
\hline \multicolumn{1}{c}{ Destino } & Porcentaje (\%) \\
\hline Combustible & 16.6 \\
Trozos y chapas & 10.6 \\
Pula & 53.6 \\
Astillas y partículas & 15.5 \\
Aserrada & 2.4 \\
Tableros & 1.4 \\
\hline
\end{tabular}

Tabla 2. Aprovechamiento de la producción de madera en Uruguay a 2010.

La explotación forestal en bosques genera entre 10-15\% de residuos; como se encuentran en una zona natural, el $2 \%$ de éstos se dejan sobre el suelo para transferencia de nutrientes. En la explotación industrial, se obtiene entre $40-50 \%$ de residuos, ya que existe una alta pérdida por la elaboración de elementos de tamaño específico; por ejemplo, la elaboración de un palo de escoba se realiza con un rectángulo, perdiendo cerca de 21,5\% (Castioglini, 2013).

\section{Aprovechamiento de residuos orgánicos}

En el campo internacional, los métodos más usados para el manejo de residuos orgánicos en general lo comprenden el compostaje y vermicompostaje, tal como se mencionó anteriormente. El compostaje se conoce como una técnica, en la cual se promueve la biodegradación de la materia orgánica por la acción de microorganismos, generando la transformación de ésta en otras formas químicas que forman el compost (Sztern y Pravia, 2001). El sistema de compostaje consiste en la formación de pilas con los residuos, con dimensiones aproximadas de 3,0 $\mathrm{m}$ de ancho, 2,4 m de largo y 1,5 m de alto, las cuales son sometidas a continuos volteos o movimiento con el fin de favorecer la entrada de oxígeno y evitar condiciones aerobias, tal como se indica en la figura 6 (Sztern, D y Pravia, 2001).

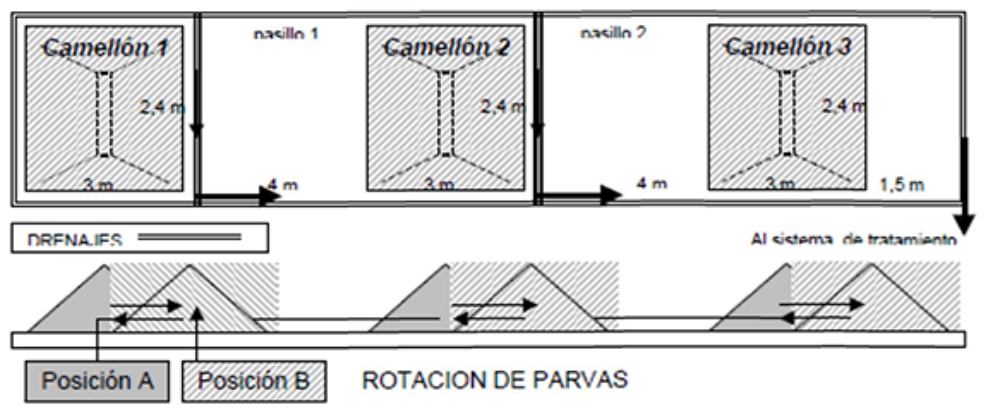

Figura 6. Dimensiones de las pilas de compostaje y sistema de volteo o rotación. Fuente:

Sztern, D y Pravia, 2001. 
Dentro del sistema se cuenta con algunos materiales que no sufren descomposición, es decir, contaminación por parte de elementos inertes, así como pérdida de volumen por la deshidratación, entre un $10-20 \%$ del volumen inicial, debido a los procesos bioquímicos y de manipulación; y un $10-20 \%$ en el cernido final para la obtención de compost (Sztern, D y Pravia, 2001). El compost es un material inocuo, es decir, no contiene patógenos ni microorganismos nocivos, ya que dentro de las fases de degradación se presenta un período termofílico, donde se alcanzan los $70^{\circ} \mathrm{C}$ aproximadamente, generando un proceso de pasteurización. En general, las cuatro fases del proceso están asistidas por diferentes microorganismos que cumplen roles complementarios para producir un elemento de calidad, con altos niveles de materia orgánica y otros elementos en formas asimilables para las plantas (Sztern y Pravia, 2001).

Para el caso del vermicompostaje, también conocido como lombricultura o alternativa biotécnica, se usa la lombriz para la transformación de los materiales orgánicos, dando como resultado humus o abono orgánico. Este sistema consta de camas usualmente con un tamaño rectangular para facilitar su manejo con dimensiones de 1,0-2,0 $\mathrm{m}$ de ancho, 0,4-0,5 $\mathrm{m}$ de alto y 2,0 m de largo; un ejemplo lo encontramos en la figura 7 (Sagarpa, 2009; Durán, L. y Henríquez, C. 2009).

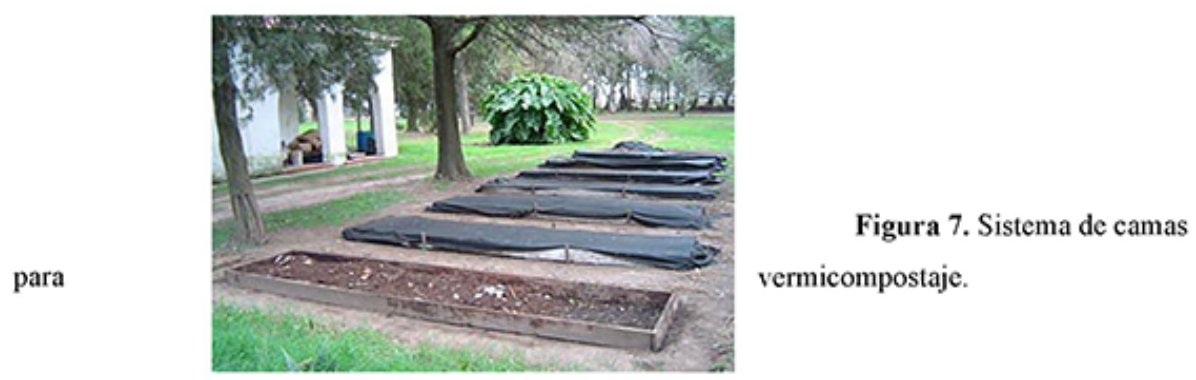

En el proceso se usan diferentes especies de lombriz, especialmente el género Eisenia; la más conocida a nivel internacional es la Roja californiana o Eisenia foétida, debido a su tolerancia para los rangos de $\mathrm{pH}$, temperatura y humedad; alta tasa reproductiva (de cada 7 a 10 días ponen una cápside o cápsula que puede contener de 2 a 21 huevos); y mayor velocidad en la degradación de los RSO; con una longevidad cercana a los 16 años (Sagarpa, 2009; Durán y Henríquez, 2009). En la tabla 3 se puede observar una producción de humus con respecto al consumo de la especie.

Tabla 3. Producción de humus por consumo.

\begin{tabular}{lllll}
\hline $\mathbf{0}$ meses & $\mathbf{3}$ meses & $\mathbf{6}$ meses & $\mathbf{9}$ meses & $\mathbf{1 2}$ meses \\
\hline Población inicial de lombrices & $1^{2}$ generación & $2^{\mathrm{a}}$ generación & $3^{\mathrm{a}}$ generación & $4^{\mathrm{a}}$ generación \\
1000 & 10.000 & 100.000 & 1.000 .000 & 10.000 .000 \\
Lombrices $1 \mathrm{~kg}$ & 10 & 100 & 1.000 & 10.000 \\
Alimento $1 \mathrm{~kg} / \mathrm{dia}$ & 10 & 100 & 1.000 & 10.000 \\
Lombricompuesto 0.6 kg/dia & 6 & 60 & 600 & 6.000 \\
Proteina $0.04 \mathrm{~kg} / \mathrm{dia}$ & 0.4 & 4 & 40 & 400 \\
\hline
\end{tabular}




\section{Conclusiones}

De acuerdo a la revisión de casos realizada, se puede afirmar que existen diferentes países capaces de proponer, implementar y mantener sistemas alternativos para la disposición de RS, RSM y RSO, de tal manera que permitan su aprovechamiento como materia prima, reintegrándolos de nuevo a la cadena productiva. En este ejercicio investigativo, se presentaron ejemplos de diferentes países con el fin de identificar la problemática actual y las posibles alternativas que se han aplicado para su solución. No obstante, son pocos los que definen y apoyan este tipo de programas, considerando el punto de vista político y legal, haciendo partícipe a la ciudadanía, de tal forma que se genere un cambio de mentalidad y comportamiento frente a la generación de estos desechos.

Países como España son los que implementan una gran variedad de alternativas en los diferentes sectores, tanto agrícolas como forestales, para la aplicación de las herramientas de la PML y la metodología de las 3R. Esto favorece la disminución de la cantidad de rellenos sanitarios necesarios y con ello la huella ambiental producida por la población. Sin embargo, países con menos desarrollo como Colombia, se encuentran atrasados en la aplicación de éstas en los diferentes sectores. Aunque a la fecha se han instaurado programas como "Basura cero" que sólo rige para la capital Bogotá, D.C., en calidad de gobierno local.

En conclusión, una vez se creen e implementen alternativas correspondientes a la disminución de residuos y aprovechamiento, como tema de interés nacional, avalado y promulgado por los gobiernos, es posible adaptar alternativas basadas en la PML, la metodología 3R y la apropiación local.

\section{Referencias}

Allevato, H. y Pórfido, D. (2002). Manejo ambiental de envases residuales de agroquímicos. Repamar, 66 pp.

Arroyave, J. y Garcés, L. (2006). Tecnologías ambientalmente sostenibles. Producción + Limpia, 1 (2), 78-86.

Bogner, J. et al. (2007). Waste Management, In Climate Change. Mitigation. Contribution of Working Group III to the Fourth Assessment Report of the Intergovernmental Panel on Climate Change [B. Metz, O.R. Davidson, P.R. Bosch, R. Dave, L.A. Meyer (eds.)], Cambridge University Press, Cambridge, United Kingdom and New York, NY, USA.

Castioglini, J. (2013). Gasificación catalítica de desechos forestales. Jornada técnica: Biomasa forestal y su uso como biocombustible. Uruguay. $50 \mathrm{pp}$.

CCA (2014). La quema de residuos agrícolas: fuente de dioxinas, Comisión para la Cooperación Ambiental, Montreal, Canadá, 6 $\mathrm{pp}$.

Cepal, FAO e IICA. (2014). "Perspectivas de la agricultura y del desarrollo rural en las Américas: una mirada hacia América Latina y el Caribe: 2014". Recuperado de www.fao. org/docrep/019/i3702s/i3702s.pdf

Comisión Económica para América Latina y el Caribe-Cepal (2013). Proyección demográfica. Observatorio Demográfico América Latina y el Caribe. Santiago de Chile. pp. 2615.

Consejería de Medio Ambiente (2012). Decreto 73/2012. Boletín Oficial de la Junta de Andalucía No. 81, pp. 74-225.

Crossette, B. (2011). "Estado de la Población Mundial 2011". Recuperado de www. unfpa.org/sites/default/files/pub-pdf/SPSWOP2011_Final.pdf.

Cuadros, S. (2008). Residuos agrícolas, forestales y lodos. EOI Escuela de Negocios, pp. 70 . 
Decreto 1713 (2002). Sobre la gestión integral de residuos sólidos. Alcaldía Mayor de Bogotá: www.alcaldiabogota.gov.co/sisjur/ normas/Norma1.jsp?i=18718\#

Decreto 4741 (2005). Sobre prevención y el manejo de los residuos o desechos peligrosos generados en el marco de la gestión integral. Alcaldía Mayor de Bogotá: www. alcaldiabogota.gov.co/sisjur/normas/Norma1 . jsp?i=18718\#.

Departamento de Desarrollo Rural y Medio Ambiente. (2010). Plan integrado de gestión de residuos de navarra y declaración de incidencia ambiental. Gobierno de Navarra. $235 \mathrm{p}$

Durán, L. y Henríquez, C. (2009). “Crecimiento y reproducción de la lombriz roja (Eisenia Foetida) en cinco sustratos orgánicos". Agronomía Costarricense, Vol. 33, Núm. 2, 2009, pp. 275-281 Recuperado de PDF/ e3ae3b5c-b104-47e9-ab80-36447537ea64.

Energy Information Administration-EIA (2008). "Annual Energy Outlook 2008". Washington, 87 p. Recuperado de www.eia. gov/oiaf/aeo/pdf/0383(2008).pdf.

Espinal, C., Martínez, H. y Peña, Y. (2005). "La cadena del plátano en Colombia. Una mirada global de su estructura y dinámica 1991-2005”. Ministerio de Agricultura y Desarrollo Rural Observatorio Agrocadenas Colombia, Documento de Trabajo No. 61. Bogotá D.C. 38 p.

Facua (2009). “Gestión de residuos domésticos y reciclaje". Recuperado de www.facua.org/ es/informe.php?Id=128\#

Food and Agriculture Organization of the United Nations-FAO (2013). FAO Statistical Yearbook. World Food and Agriculture. Rome. Recuperado de www.fao.org/docrep/018/ i3107e/i3107e00.htm.

Guajardo, P. (2010). “Hacer más”. Kimberly-
Clark. Recuperado de https://www.kimberlyclark.com.mx/informacion_financiera/anual/ Sustentabilidad.pdf

HonduPalma. (2011). Manejo de Residuos Sólidos. Tegucigalpa. México. SNV y HonduPalma.

Hoornweg y Bhada, (2012). What a waste. A global review of solid waste management. Washington. USA. World Bank.

Instituto Colombiano de Normas Técnicas y Certificación-Icontec (2009). GTC 24: Gestión Ambiental: Residuos sólidos, guía para la separación en la fuente.

Macua, J. et al. (2009). Utilización de cubiertas en el tomate de industria en Navarra. Navarra Agraria. No. 179. 29 -38.

Martínez, C. y Sandoval, H. (2015). "Autonomía energética y alimentaria de la comunidad indígena nasa mediante la gestión integral de la materia orgánica en el norte del cauca, Colombia". Fundación Pro-Orgánica. Recuperado de energiayambienteandina.net/ getattachment $/ 50 \mathrm{fb} 0 \mathrm{ac} 6-20 \mathrm{bb}-4 \mathrm{e} 31$-a $1 \mathrm{~b} 6$ 266786c5b078/Autonomia-energetica-yalimentaria-del-pueblo-Nasa.aspx

Mazzeo, M. et al. (2010). “Aprovechamiento industrial de residuos de cosecha y postcosecha del plátano en el departamento de caldas . En: Revista Educación en Ingeniería. pp. 128-139. Ministerio de Medio Ambiente y Agua-MMAA (2010). "Guía para el aprovechamiento de residuos sólidos orgánicos". 1 Ed. Bolivia. Recuperado de www.anesapa. org/wp-content/uploads/2014/12/ GuiaResiduosSolidosOrganicos.pdf

Ministerio de Agricultura, Alimentación y Medio Ambiente-Magrama (2012). Producción y Consumo Sostenible y Residuos Agrarios. Madrid. 89 p.

. (2013). Programa Estatal

de Prevención de Residuos. Recuperado 
de: $\quad$ www.magrama.gob.es/es/calidad-yevaluacion-ambiental/planes-y-estrategias/ Programa_de_prevencion_aprobado_ actualizado_ANFABRA_11_02_2014_tcm7310254.pdf

Oficina de Estudios Económicos y Estadísticos-OEEE (2011). "Producción Pecuaria e Industria Avícola 2011”. Ministerio de Agricultura. 167 p.

Organization for Economic Co-operation and Development-OECD (2000). "Working Party on Pollution Prevention and Control". Recuperado de www.oecd. org/officialdocuments/publicdisplaydocum entpdf/?doclanguage $=$ en $\&$ cote $=$ env $/$ epoc $/$ ppc\%282000\%295/final.

. (2010). Resource Productivity in the G8 and the OECD. A Report in the Framework of the Kobe 3R Action Plan. Recuperado de: www.oecd.org/env/ waste/47944428.pdf.

(2011). "Resource

Productivity in the G8 and the OECD. A Report in the Framework of the Kobe 3R Action Plan". Recuperado de www.oecd.org/ env/waste/47944428.pdf.

Pongrácz, E. y Pohjola, V. (2002). “An approach to the formal theory of waste management. Resources, Conservation and Recycling", 35, 17-29.

(2004). Re-defining waste, the concept of ownership and the role of waste management. Resources, Conservation and Recycling, 40, 141-153.

Population Reference Bureau-PRB. (2013). "Cuadro de Datos de la Población Mundial". Recuperado de www.prb.org/pdf14/2013population-data-sheet_spanish.pdf.

Programa de las Naciones Unidas para el Medio Ambiente-PNUMA. (2013). "Tendencias del flujo de materiales y productividad de recursos en América Latina". Recuperado de www. pnuma.org/MFA_espanol\%201ow\%20res.pdf.

"Estados del Medio

Ambiente y Medidas Normativas: 19722002”. Recuperado de www.grida.no/geo/ geo3/spanish/pdfs/chapter2-8_urban.pdf

Sagarpa (2009). “Abonos orgánicos: Lombricultura". Secretaría de Agricultura, Ganadería, Desarrollo rural, Pesca y Alimentación. México. 8 p.

Sandach (2015). "Legislación Subproductos". Recuperado de aesan.msssi.gob.es/AESAN/ web/legislacion/subdetalle/subproductos. shtml.

Secretaría de Gobierno-SeGob. (2014). Programa Nacional Forestal 2014-2018. México. Recuperado de www.dof.gob.mx/ nota_detalle.php?codigo $=5342498 \& \mathrm{fec}$ $h a=28 / 04 / 2014$

Semarnat (2008). Programa Estratégico Forestal del Estado de Oaxaca (PEFO) 2007-2030. México. Recuperado de www.conafor.gob.mx:8080/ documentos/docs/12/187Programa $\% 20$ Estrat\%C3\%A9gico\%20Forestal\%20del\%20 Estado\%20de\%20Oaxaca.pdf

Sigfito (2012). "Programa Sigfito". Recuperado de sigfito.es/

Steffen, W. et al. (2004). Global Change and the Earth System. Heidelberg. Alemania. IGBP Secretariat Royal Swedish Academy of Sciences.

Sztern, D. y Pravia, M. (2001). "Manual para la Elaboración de Compost. Bases Conceptuales y Procedimientos". Organización Panamericana dela Salud. Recuperadode www.ambiente.gov. ar/archivos/web/PNGIRSU/file/Documentos/ Bases $\% 20$ conceptuales $\% 20$ para $\% 201$ a $\% 20$ elaboracion $\% 20 \mathrm{de} \% 20$ compost.pdf

Tello, P. et al. (2011). "Informe de la 
Evaluación Regional del Manejo de Residuos Sólidos Urbanos en ALC 2010”. Organización Panamericana de la Salud (OPS), Banco Interamericano de Ingeniería Sanitaria $\mathrm{Y}$ Ambiental (AIDIS). Banco Interamericano de Desarrollo (BID), pp. 164 Recuperado de idbdocs.iadb.org/wsdocs/getdocument. aspx?docnum $=36466973$

Unidad Administrativa Especial de Servicios Públicos-UAESP (2010). "Programa para la gestión de los residuos sólidos orgánicos para la ciudad de Bogotá, D.C. Bogotá, pp. 322.

Environmental Protection Agency-EPA (2014). "Municipal Solid Waste Generation, Recycling, and Disposal in the United States: Facts and Figures for 2012". Recuperado de www.epa.gov/wastes/nonhaz/municipal/ pubs/2012_msw_fs.pdf

Velázquez Martí, B. (2006). "Situación de los sistemas de aprovechamiento de los residuos forestales para su utilización energética". En: Revista Científica y Técnica de Ecología y Medio Ambiente. Vol. 15, No. 1, pp. 77-86. 